\title{
On the intersection product of analytic cycles
}

\author{
by SŁaWOmir Rams (Kraków)
}

\begin{abstract}
We prove that the generalized index of intersection of an analytic set with a closed submanifold (Thm. 4.3) and the intersection product of analytic cycles (Thm. 5.4), which are defined in $\left[\mathrm{T}_{2}\right]$, are intrinsic. We define the intersection product of analytic cycles on a reduced analytic space (Def. 5.8) and prove a relation of its degree and the exponent of proper separation (Thm. 6.3).
\end{abstract}

1. Introduction. In $\left[\mathrm{T}_{2}\right]$ an intersection product of two analytic cycles on a (complex) manifold is constructed. The construction is based on a pointwise defined so-called extended index of intersection $\widetilde{g}(Z, S)(c)$ of an analytic subset $Z$ of a manifold $N$ with a submanifold $S$ at a point $c$ (see $\left[\mathrm{T}_{2}\right]$, p. 185). This notion generalizes the intersection product constructed in $[\mathrm{Dr}],[\mathrm{BH}]$, and the isolated improper intersection multiplicity studied in $[\mathrm{ATW}]$.

This paper is a continuation of $\left[\mathrm{T}_{2}\right]$ and its main aim is to prove that both the extended index of intersection and the intersection product are intrinsic. Thanks to the results of the first five sections of our paper the construction from $\left[\mathrm{T}_{2}\right]$ can be applied to analytic cycles on reduced analytic spaces (Def. 5.8), which leads to a generalization (Thm. 6.3) of some estimates of the exponent of proper separation (see $\left[\mathrm{T}_{1}\right],[\mathrm{CT}],[\mathrm{Cg}]$ ).

The reader is expected to be familiar with the notion of the multiplicity of proper intersection of analytic sets (see $[\mathrm{Dr}]$ ), intersection product of analytic cycles (see $\left[\mathrm{T}_{2}\right]$ ), and some properties of holomorphic cycles (also known as chains) (see $\left[T_{2}, \S 3\right]$ ). For the convenience of the reader we present the construction of the intersection product of analytic cycles on a manifold in the third section. The definition of the topology of chains can be found in $\left[\mathrm{R}_{1}\right]$. We apply it only on the space of positive chains, where it coincides with

2000 Mathematics Subject Classification: 32B15, 32C25, 32C30, 32C99.

Key words and phrases: improper intersection, extended index of intersection, regular separation.

Partially supported by KBN Grant 2 P03A 00115. 
the weak topology of currents. Throughout the text we work over the field $\mathbb{C}$ of complex numbers. All the manifolds are assumed to be second-countable.

2. Preliminaries. Let $N$ be an $n$-dimensional complex manifold, $M$ be an $m$-dimensional closed submanifold of $N$, and let $S$ be an $s$-dimensional closed submanifold of $M$. We fix a purely $k$-dimensional analytic set $Z \subset M$ and a point $c \in Z \cap S$. Let $U$ be a neighbourhood of $c$ in $N$.

We define the following family of hypersurfaces:

$\mathcal{B}(U):=\{H \subset U: H$ is a smooth hypersurface in $U, H \supset S \cap U\}$.

We endow $\mathcal{B}(U)$ with the topology of $(n-1)$-chains (see $\left.\left[\mathrm{R}_{1}\right]\right)$ and obtain a metrizable topological space $\left(\left[\mathrm{R}_{1}\right]\right.$, Thm. 3.6).

Let us recall some basic notions associated with analytic cycles. Let $A=\sum_{j \in J} \alpha_{j} C_{j}$ be an analytic cycle on $N$. The degree of such a cycle at a point $c$ is defined to be the sum $\sum_{j \in J} \alpha_{j} \nu\left(C_{j}, c\right)$, where $\nu\left(C_{j}, c\right)$ stands for the degree of the component $C_{j}$ at $c$. By [ $\left.\mathrm{T}_{2}\right]$, Prop. 2.1, the function $N \ni c \mapsto \nu(A, c) \in \mathbb{Z}$ is analytically constructible, and for each analytically constructible function $f: N \rightarrow \mathbb{Z}$ there exists a unique cycle whose degree equals the value of $f$ at every point of $N$. This cycle will be called the cycle defined by the function $f$.

The analytic cycle $A$ has a unique decomposition into the sum of $j$-cycles (formal combinations of analytic sets of pure dimension $j$ ) $A=\sum_{j=0}^{n} T_{(j)}$. The extended degree of $A$ at $c$ is defined by the formula

$$
\widetilde{\nu}(A, c):=\left(\nu\left(T_{(n)}, c\right), \ldots, \nu\left(T_{(0)}, c\right)\right) \in \mathbb{Z}^{n+1} .
$$

For the cycle $A=\sum_{j \in J} \alpha_{j} C_{j}$ the part of $A$ supported by $S$ is defined to be

$$
A^{S}:=\sum_{j \in J, C_{j} \subset S} \alpha_{j} C_{j} .
$$

Let $V$ be an open subset of $N$. The restriction of the cycle $A$ to $V$ is defined to be $\sum_{j \in J} \alpha_{j}\left(C_{j} \cap V\right)$ and denoted by $A \cap V$.

Throughout the text $E$ denotes the unit disc in $\mathbb{C}$. We end this section with the following useful lemma.

Lemma 2.1. Let $N=E^{n}, S=E^{s} \times\{0\}^{n-s}$, and let $\left\{F_{\nu}\right\}_{\nu=1}^{\infty}$ be a sequence of orthogonal mappings such that each $F_{\nu}$ is $\operatorname{id}_{\mathbb{C}^{s}} \oplus f_{\nu}$, where $f_{\nu}$ : $\mathbb{C}^{n-s} \rightarrow \mathbb{C}^{n-s}$. If $F_{\nu} \rightarrow \mathrm{id}_{\mathbb{C}^{n}}$ and a hypersurface $H$ belongs to $\mathcal{B}\left(E^{n}\right)$, then $F_{\nu}(H) \rightarrow H$.

Proof. This is a straightforward consequence of $\left[\mathrm{T}_{2}\right]$, Lemma 3.2.

3. Intersections on manifolds. In this section we maintain the setup of the previous one. We recall here some basic facts from $\left[\mathrm{T}_{2}\right]$ and study 
the behaviour of the systems of hypersurfaces that appear in $\left[\mathrm{T}_{2}\right]$, Algorithm (4.1).

Let us recall the definition of the family $\mathcal{H}(U, Z)$ (see $\left.\left[\mathrm{T}_{2}\right], \mathrm{p} .184\right)$. It is the family of all $\mathcal{H}:=\left(H_{1}, \ldots, H_{n-s}\right)$ satisfying the following conditions:

(a) $H_{j}$ is a smooth hypersurface of $U$ containing $U \cap S$ for $j=1, \ldots, n-s$,

(b) $\bigcap_{j=1}^{n-s} T_{x} H_{j}=T_{x} S$ for each $x \in U \cap S$,

(c) $\operatorname{dim}\left[((U \backslash S) \cap Z) \cap H_{1} \cap \ldots \cap H_{j}\right] \leq \max \{k-j,-1\}$ for $j=0,1, \ldots, k$.

The family $\mathcal{H}(U, Z)$ is also denoted by $\mathcal{H}_{S}(U, Z)$.

Any system $\mathcal{H}:=\left(H_{1}, \ldots, H_{n-s}\right) \in \mathcal{H}(U, Z)$ enables us to construct an analytic cycle $\mathcal{H} \cdot Z$ in $S \cap U$ by the following procedure:

Algorithm ((4.1) from $\left.\left[\mathrm{T}_{2}\right]\right)$.

STEP 0: Let $Z_{0}=Z \cap U$. Then $Z_{0}=Z_{0}^{S}+\left(Z_{0}-Z_{0}^{S}\right)$, where $Z_{0}^{S}$ is the part of $Z_{0}$ supported by $S \cap U$ (see [T $\left.\mathrm{T}_{2}\right]$, p. 182).

Step 1: Let $Z_{1}=\left(Z_{0}-Z_{0}^{S}\right) \cdot H_{1}$. Then $Z_{1}=Z_{1}^{S}+\left(Z_{1}-Z_{1}^{S}\right)$, where $Z_{1}^{S}$ is the part of $Z_{1}$ supported by $S \cap U$.

STEP 2: Let $Z_{2}=\left(Z_{1}-Z_{1}^{S}\right) \cdot H_{2}$. Then $Z_{2}=Z_{2}^{S}+\left(Z_{2}-Z_{2}^{S}\right)$, where $Z_{2}^{S}$ is the part of $Z_{2}$ supported by $S \cap U$.

STEP $n-s$ : Let $Z_{n-s}=\left(Z_{n-s-1}-Z_{n-s-1}^{S}\right) \cdot H_{n-s}$. Now we have a decomposition $Z_{n-s}=Z_{n-s}^{S}+\left(Z_{n-s}-Z_{n-s}^{S}\right)$, where $\left|Z_{n-s}-Z_{n-s}^{S}\right|$ $\cap S=\emptyset$.

The cycle $\mathcal{H} \cdot Z:=Z_{0}^{S}+\ldots+Z_{n-s}^{S}$ is called the result of the Algorithm applied to the system $\mathcal{H}$, the set $Z$ and the submanifold $S$.

We define the extended index of intersection $\widetilde{g}(Z, S)(c)$ of $Z$ and $S$ at $c$ by the formula ([T $\left.\mathrm{T}_{2}\right]$, Def. 4.2)

$\widetilde{g}(Z, S)(c):=\min _{\operatorname{lex}}\{\widetilde{\nu}(\mathcal{H} \cdot Z, c): \mathcal{H} \in \mathcal{H}(V, Z), V$ is a neighbourhood of $c\}$, where lex denotes the lexicographical ordering. The sum of all elements of $\widetilde{g}(Z, S)(c)$ is called the index of intersection of $Z$ and $S$ at $c$ and is denoted by $g(c)\left(\right.$ or $\left.g_{N}(Z, S)(c)\right)$.

Let $X, Y$ be irreducible analytic subsets of the manifold $N, a \in N$, and let $\Delta_{N}$ denote the diagonal in $N^{2}$. By $\left[\mathrm{T}_{2}\right]$, Thm. 6.2, the function

$$
N \ni x \mapsto g_{N^{2}}\left(X \times Y, \Delta_{N},(x, x)\right) \in \mathbb{N}
$$

is analytically constructible. The cycle defined by this function is called the intersection product of the sets $X, Y$ and is denoted by $X \bullet Y$.

Having presented the construction which plays a fundamental role in this paper, we can introduce some useful notation. Given a system $\left(H_{1}, \ldots, H_{p}\right)$ of hypersurfaces and an open set $V$, we define a system of locally analytic sets by the equality

$$
\left.\left(H_{1}, \ldots, H_{p}\right)\right|_{V}:=\left(H_{1} \cap V, \ldots, H_{p} \cap V\right) .
$$


Let us now fix an integer $1 \leq i \leq n-s$ and a system $\mathcal{H}=\left(H_{1}, \ldots, H_{n-s}\right)$ of elements of $\mathcal{B}(U)$. For $H \in \mathcal{B}(U)$ we define the system

$$
\mathcal{H}_{i}(H):=\left(H_{1}, \ldots, H_{i-1}, H, H_{i+1}, \ldots, H_{n-s}\right) .
$$

$\mathcal{D}(U, Z)$ denotes the family of all $\mathcal{H}=\left(H_{1}, \ldots, H_{n-s}\right)$, where $H_{j} \in \mathcal{B}(U)$, which satisfy the condition

$$
\left(\mathrm{s}_{q}\right) \quad \operatorname{dim}\left[((U \backslash S) \cap Z) \cap H_{1} \cap \ldots \cap H_{q}\right] \leq \max \{k-q,-1\}
$$

for $q=1, \ldots, k$.

Observe that for any system $\mathcal{H}=\left(H_{1}, \ldots, H_{n-s}\right) \in \mathcal{D}(U, Z)$ we can construct the cycle $\mathcal{H} \cdot Z$. If $V$ is a neighbourhood of $c$ and $\left.\mathcal{H}\right|_{V} \in \mathcal{D}(V, Z)$, then we denote $\widetilde{\nu}\left(\left(\left.\mathcal{H}\right|_{V} \cdot Z\right), c\right)$ by $\widetilde{\nu}(\mathcal{H} \cdot Z, c)$. This notation is justified by the fact that the extended degree of the cycle $\left.\mathcal{H}\right|_{V} \cdot Z$ at $c$ depends only on the germs of $Z, S, H_{1}, \ldots, H_{n-s}$ at $c$. The latter results from the equality $\left(\left.\mathcal{H}\right|_{V} \cdot Z\right) \cap V_{1}=\left.\mathcal{H}\right|_{V_{1}} \cdot Z$, which holds for any neighbourhood $V_{1} \subset V$ of $c$.

In what follows we will need the following families of hypersurfaces: $\mathcal{I}_{i}(\mathcal{H}, U, Z)$ denotes the family of hypersurfaces $H \in \mathcal{B}(U)$ for which there exists a neighbourhood $V$ of $c$ such that $\left.\mathcal{H}_{i}(H)\right|_{V} \in \mathcal{H}(V, Z)$, and

$$
\mathcal{J}_{i}(\mathcal{H}, U, Z):=\left\{H \in \mathcal{I}_{i}(\mathcal{H}, U, Z): \widetilde{\nu}\left(\mathcal{H}_{i}(H) \cdot Z, c\right)=\widetilde{g}(Z, S)(c)\right\} .
$$

Lemma 3.1. Let $\mathcal{H}=\left(H_{1}, \ldots, H_{n-s}\right) \in \mathcal{H}(U, Z)$ and let $\left\{H_{i}^{j}\right\}_{j=1}^{\infty} \subset$ $B(U)$ converge to $H_{i}^{0}$. If $H_{i}^{0} \in \mathcal{J}_{i}(\mathcal{H}, U, Z)$ then there exists a neighbourhood $V$ of $c$ such that for almost all $j$ we have

$$
\left.\mathcal{H}_{i}\left(H_{i}^{j}\right)\right|_{V} \in \mathcal{D}(V, Z) \text {. }
$$

Proof. If $\widetilde{U}$ is a neighbourhood of $c$ such that the system $\left.\mathcal{H}_{i}\left(H_{i}^{j}\right)\right|_{\widetilde{U}}$ satisfies $\left(\mathrm{s}_{q}\right)$ for $j=q, \ldots, l$, then $Z_{l}(j, \widetilde{U}), Z_{l}^{S}(j, \widetilde{U})$ stand for the results of the first $l$ steps of the Algorithm applied to this system.

It is obvious that all $\mathcal{H}_{i}\left(H_{i}^{j}\right)$ satisfy $\left(\mathrm{s}_{q}\right)$ for $q<i$. By $\left[\mathrm{TW}_{1}\right]$, Thm. 2 , there exists a neighbourhood $U_{i}$ of $c$ and an integer $j_{i}$ such that $H_{i}^{j} \cap U_{i}$ meet $Z \cap U_{i}$ properly for $j>j_{i}$, which means that the cycles $Z_{i}\left(j, U_{i}\right)$ are well defined for $j>j_{i}$.

We can apply $\left[\mathrm{T}_{2}\right]$, Thm. 3.6, to obtain $Z_{i}\left(j, U_{i}\right) \rightarrow Z_{i}\left(0, U_{i}\right)$. By $\left[\mathrm{T}_{2}\right]$, Thm. 3.4, there exists a neighbourhood $\widetilde{U}_{i+1} \subset U_{i}$ of $c$ such that

$$
\left(Z_{i}\left(j, U_{i}\right)-Z_{i}^{S}\left(j, U_{i}\right)\right) \cap \widetilde{U}_{i+1} \rightarrow\left(Z_{i}\left(0, U_{i}\right)-Z_{i}^{S}\left(0, U_{i}\right)\right) \cap \widetilde{U}_{i+1} .
$$

Since $\nu\left(\left(Z_{i}\left(0, U_{i}\right)-Z_{i}^{S}\left(0, U_{i}\right)\right)^{H_{i+1}}, c\right)=0$, we can apply $\left[\mathrm{T}_{2}\right]$, Thm. 3.4, to find a neighbourhood $\widehat{U}_{i+1} \subset \widetilde{U}_{i+1}$ such that

$$
\left(\left(Z_{i}\left(j, U_{i}\right)-Z_{i}^{S}\left(j, U_{i}\right)\right)^{H_{i+1}}\right) \cap \widehat{U}_{i+1} \rightarrow 0,
$$

where 0 is the zero cycle in $\widehat{U}_{i+1}$. Consequently, there exists a neighbourhood $U_{i+1} \subset \widehat{U}_{i+1}$ of $c$ and an integer $j_{i+1}$ such that $Z_{i+1}\left(j, U_{i+1}\right)$ is well defined for $j>j_{i+1}$. 
Since $Z_{i+1}\left(j, U_{i+1}\right)=\left(H_{i+1} \cap U_{i+1}\right) \cdot\left(Z_{i}\left(j, U_{i+1}\right)-Z_{i}^{S}\left(j, U_{i+1}\right)\right)$ we apply $\left[\mathrm{T}_{2}\right]$, Thm. 3.6, again to obtain the convergence $Z_{i+1}\left(j, U_{i+1}\right) \rightarrow$ $Z_{i+1}\left(0, U_{i+1}\right)$. Consequently, this procedure can be repeated until we find a neighbourhood $U_{n-s}$ of $c$ and an integer $j_{n-s}$ such that the cycles $Z_{n-s}\left(j, U_{n-s}\right)$ are well defined for $j>j_{n-s}$. Put $V:=U_{n-s}$.

We can now prove the following property of the family $\mathcal{J}_{i}(\mathcal{H}, U, Z)$.

Proposition 3.2. Under the main assumptions of this section we have

$$
\mathcal{J}_{i}(\mathcal{H}, U, Z) \subset \operatorname{Int}\left(\mathcal{I}_{i}(\mathcal{H}, U, Z)\right),
$$

where $\mathcal{I}_{i}(\mathcal{H}, U, Z)$ is considered as a subset of the space $\mathcal{B}(U)$.

P r o of. Let $\breve{H}_{i} \in \mathcal{J}_{i}(\mathcal{H}, U, Z)$. It suffices to find a neighbourhood $\mathcal{V}$ of $\breve{H}_{i}$ in $\mathcal{B}(U)$ such that every $H_{i} \in \mathcal{V}$ satisfies the following conditions:

(a) $\bigcap_{j=1}^{n-s} T_{c} H_{j}=T_{c} S$,

(b) there exists a neighbourhood $\widetilde{U}$ of $c$ such that

$$
\operatorname{dim}\left[(Z \cap(\widetilde{U} \backslash S)) \cap H_{1} \cap \ldots \cap H_{j}\right] \leq \max \{k-j,-1\} \quad \text { for } j \leq k .
$$

To find a neighbourhood consisting only of hypersurfaces satisfying (a) we can assume that $U=(1+\varepsilon) E^{n}$, where $\varepsilon>0, S=(1+\varepsilon) E^{s} \times\{0\}^{n-s}$, $c=0$. Observe that $\bigcap_{j \neq i} T_{0} H_{j}=T_{0} S+l$, where $l$ is a line.

Choose $v \in\left(T_{0} S+l\right) \backslash T_{0} S$. According to $\left[\mathrm{R}_{1}\right]$, Def. 2.4, the condition $v \notin T_{0} H$ defines a neighbourhood of $T_{0} \breve{H}_{i}$. Therefore, it suffices to prove the continuity of the mapping

$$
\mathcal{B}(U) \ni H \mapsto T_{0} H \in \mathcal{B}(U) .
$$

Let $\left\{H^{\nu}\right\}_{\nu=0}^{\infty} \subset \mathcal{B}(U)$ converge to a hypersurface $H^{0}$. Without loss of generality $H^{0}=(1+\varepsilon) E^{n-1} \times\{0\}$. For sufficiently large $\nu$ we have

$$
\left(\overline{E^{n-1}} \times \partial E\right) \cap H^{\nu}=\emptyset \text { and } \mu\left(\left.\pi\right|_{H^{\nu} \cap E^{n}}\right)=1 \text {, }
$$

where $\pi\left(x_{1}, \ldots, x_{n}\right):=\left(x_{1}, \ldots, x_{n-1}\right)$ and $\mu$ denotes the multiplicity of holomorphic covering.

Consequently, almost all hypersurfaces $H^{\nu} \cap E^{n}$ are the graphs of holomorphic mappings $G_{\nu}: E^{n-1} \rightarrow E$, and $G_{\nu}$ converges uniformly to $G_{0}$. The latter fact yields the convergence $G_{\nu}^{\prime}(0) \rightarrow G_{0}^{\prime}(0)$ and we get $T_{0} H^{\nu} \rightarrow T_{0} H^{0}$.

The existence of a neighbourhood satisfying (b) results immediately from Lemma 3.1.

Now we can prove the main theorem of this section.

THEOREM 3.3. The family $\mathcal{J}_{i}(\mathcal{H}, U, Z)$ is an open subset of $\mathcal{B}(U)$.

P r o of. By the previous proposition it suffices to prove that $\mathcal{J}_{i}(\mathcal{H}, U, Z)$ is an open subset of $\mathcal{I}_{i}(\mathcal{H}, U, Z)$. Suppose that there exists a sequence $\left\{H^{j}\right\}_{j=1}^{\infty} \subset \mathcal{I}_{i}(\mathcal{H}, U, Z) \backslash \mathcal{J}_{i}(\mathcal{H}, U, Z)$ which converges to $H^{0} \in \mathcal{J}_{i}(\mathcal{H}, U, Z)$. 
We introduce the following notation: $Z_{l}(j), Z_{l}^{S}(j)$ stand for the results of the $l$ th step of the Algorithm applied to the systems $\mathcal{H}_{i}\left(H^{j}\right)$ (thanks to Lemma 3.1 we can assume that all those cycles are well defined), and

$$
\left(\nu_{s}^{j}, \ldots, \nu_{0}^{j}\right):=\widetilde{\nu}\left(\mathcal{H}_{i}\left(H^{j}\right) \cdot Z, c\right), \quad \alpha:=\max \left\{l: \lim \sup \nu_{l}^{j}>\nu_{l}^{0}\right\} .
$$

Obviously $Z_{q}^{S}(j)=Z_{q}^{S}(0)$ for $q \leq i-1$ and $\nu_{l}^{j}=\nu_{l}^{0}$ for $l \geq k-i+1$. According to $\left[\mathrm{T}_{2}\right]$, Thm. 3.6, we have $Z_{1}(j) \rightarrow Z_{1}(0)$. By $\left[\mathrm{T}_{2}\right]$, Thm. 3.4, there exists a neighbourhood $U_{i}$ of $c$ such that

$$
\begin{aligned}
Z_{i}^{S}(j) & \cap U_{i} \rightarrow Z_{i}^{S}(0) \cap U_{i}, \\
\left(Z_{i}(j)-Z_{i}^{S}(j)\right) \cap U_{i} & \rightarrow\left(Z_{i}(0)-Z_{i}^{S}(0)\right) \cap U_{i} .
\end{aligned}
$$

The convergence $\left(\mathrm{a}_{i}\right)$ yields that $\lim \sup \nu_{i}^{j}=\nu_{i}^{0}$, which gives $\alpha<i$. Since $H^{0} \in \mathcal{J}_{i}(\mathcal{H}, U, Z)$ and $\left(\mathrm{b}_{i}\right)$ holds, we can apply $\left[\mathrm{T}_{2}\right]$, Thm. 3.4, to find $U_{i+1}$ satisfying the conditions $\left(\mathrm{a}_{i+1}\right),\left(\mathrm{b}_{i+1}\right)$. Finally, we get $\alpha<0$, which contradicts our choice of $\left\{H^{j}\right\}_{j=1}^{\infty}$.

4. Extended index of intersection. We keep the setup of the previous section. In this section we prove that the extended index of intersection is intrinsic.

We will need the following lemmas.

Lemma 4.1. Let $N=E^{n}, M=E^{m} \times\{0\}^{n-m}, S=E^{s} \times\{0\}^{n-s}$ and let $Z$ be a purely $k$-dimensional analytic subset of $M$. If $V$ is a neighbourhood of 0 in $M$, and $\mathcal{H}=\left(H_{1}, \ldots, H_{m-s}\right) \in \mathcal{H}(V, Z)$, then there exist hypersurfaces $H_{m-s+1}, \ldots, H_{n-s}$ such that the system

$$
\widetilde{\mathcal{H}}:=\left(H_{1} \times E^{n-m}, \ldots, H_{m-s} \times E^{n-m}, H_{m-s+1}, \ldots, H_{n-s}\right)
$$

belongs to $\mathcal{H}\left(V \times E^{n-m}, Z\right)$, and $\widetilde{\nu}(\widetilde{\mathcal{H}} \cdot Z, 0)=\widetilde{\nu}(\mathcal{H} \cdot Z, 0)$.

Proof. Let $Z_{j}, Z_{j}^{S}$ (resp. $\widetilde{Z}_{j}, \widetilde{Z}_{j}^{S}$ ) denote the result of the $j$ th step of the Algorithm applied to $\mathcal{H}$ (resp. $\widetilde{\mathcal{H}}$ ), $Z$ and $S$.

Choose a system of hyperplanes $H_{m-s+1}, \ldots, H_{n-s}$ such that $\mathbb{C}^{s} \times\{0\}^{m-s}$ $\times \mathbb{C}^{n-m} \cap \bigcap_{j=m-s+1}^{n-s} H_{j}=\mathbb{C}^{s} \times\{0\}^{n-s}$. The assumption $Z \subset M$ gives

$$
\bigcap_{j=1}^{m-s}\left(H_{j} \times E^{n-m}\right) \cap Z \subset\left(\bigcap_{j=1}^{m-s} H_{j}\right) \times\{0\}^{n-m}=S,
$$

which implies that $\widetilde{\mathcal{H}} \in \mathcal{H}\left(V \times E^{n-m}, Z\right)$ and the cycles $\widetilde{Z}_{j}^{S}$ vanish for $j>m-s$.

It suffices to prove the equality $Z_{j}=\widetilde{Z}_{j}$ for $j \leq m-s$. We can assume that $V=M$ and proceed by induction on $j$. Obviously $Z_{0}=\widetilde{Z}_{0}$. Suppose that $Z_{j-1}=\widetilde{Z}_{j-1}$. Then [TW $]$, Thm. 2.2, yields

$$
\left(H_{j} \times E^{n-m}\right) \cdot{ }_{N}\left(\widetilde{Z}_{j-1}-\widetilde{Z}_{j-1}^{S}\right)=\left(\left(H_{j} \times E^{n-m}\right) \cdot_{N} M\right) \cdot{ }_{M}\left(\widetilde{Z}_{j-1}-\widetilde{Z}_{j-1}^{S}\right) .
$$


Since $\left(H_{j} \times E^{n-m}\right) \cdot{ }_{N} M=H_{j}$ and $\widetilde{Z}_{j-1}-\widetilde{Z}_{j-1}^{S}=Z_{j-1}-Z_{j-1}^{S}$ we get $Z_{j}=\widetilde{Z}_{j}$

LEMMA 4.2. Under the assumptions of Lemma 4.1, if $U$ is a neighbourhood of 0 in $N$, and $\widetilde{\mathcal{H}}=\left(\widetilde{H}_{1}, \ldots, \widetilde{H}_{n-s}\right) \in \mathcal{H}(U, Z)$ satisfies the condition

$$
\bigcap_{i=1}^{m-s} T_{0} \widetilde{H}_{i} \cap M=S,
$$

then there exists a neighbourhood $V$ of 0 in $\underset{\widetilde{\mathcal{H}}}{M}$ such that the system $\mathcal{H}:=$ $\left.\left(\widetilde{H}_{1} \cdot M, \ldots, \widetilde{H}_{m-s} \cdot M\right)\right|_{V} \in \mathcal{H}(V, Z)$ and $\widetilde{\nu}(\widetilde{\mathcal{H}} \cdot Z, 0)=\widetilde{\nu}(\mathcal{H} \cdot Z, 0)$.

P r o of. One can easily see that $\left.\left(\widetilde{H}_{1} \cdot M, \ldots, \widetilde{H}_{m-s} \cdot M\right)\right|_{V} \in \mathcal{H}(V, Z)$ for a sufficiently small $V$.

Let $Z_{j}, Z_{j}^{S}$ (resp. $\widetilde{Z}_{j}, \widetilde{Z}_{j}^{S}$ ) stand for the result of the $j$ th step of the Algorithm applied to $\mathcal{H}$ (resp. $\widetilde{\mathcal{H}}$ ), $Z$ and $S$. As in the proof of Lemma 4.1 we have the inclusion $\bigcap_{j=1}^{m-s} H_{j} \cap Z \subset S$, which shows that the cycles $\widetilde{Z}_{j}^{S}$ vanish for $j>m-s$.

Finally, [TW $\mathrm{TW}_{2}$, Thm. 2.2, yields $Z_{j}=\widetilde{Z}_{j} \cap(V \cap S)$ for $j \leq m-s$.

THEOREM 4.3. Let $S$ be an s-dimensional closed submanifold of a manifold $M$, and let $Z$ be a purely $k$-dimensional analytic subset of $M, c \in S$. If $M$ is a submanifold of a manifold $N$, then

$$
\widetilde{g}_{N}(Z, S)(c)=\widetilde{g}_{M}(Z, S)(c) .
$$

Proof. Without loss of generality $N=E^{n}, M=E^{m} \times\{0\}^{n-m}, S=$ $E^{s} \times\{0\}^{n-s}, c \in Z \cap S$ and $c=0$. We can also assume that there exists a system $\mathcal{H}=\left(H_{1}, \ldots, H_{n-s}\right) \in \mathcal{H}(N, Z)$ such that $\widetilde{\nu}(\mathcal{H} \cdot Z, c)=\widetilde{g}(Z, S)(c)$.

We construct $\mathcal{H}^{m-s}:=\left(\widetilde{H}_{1}, \ldots, \widetilde{H}_{m-s}, H_{m-s+1}, \ldots, H_{n-s}\right)$ and find $\varepsilon_{q}>0$ such that $\mathcal{H}^{m-s}$ satisfies (1) (see Lemma 4.2 ), $\left.\mathcal{H}^{m-s}\right|_{\varepsilon_{m-s} E^{n}}$ belongs to $\mathcal{H}\left(\varepsilon_{m-s} E^{n}, Z\right)$ and $\widetilde{\nu}\left(\mathcal{H}^{m-s} \cdot Z, c\right)=\widetilde{g}(Z, S)(c)$.

Construction of $\widetilde{H}_{1}$. If $H_{1}$ meets $M$ at 0 transversally, we put $\widetilde{H}_{1}:=H_{1}$. Otherwise we choose $\left\{F_{\nu}\right\}_{\nu=1}^{\infty}$ satisfying the following conditions:

(a) $F_{\nu}=\operatorname{id}_{\mathbb{C}^{s}} \oplus f_{\nu}$, where $f_{\nu}: \mathbb{C}^{n-s} \rightarrow \mathbb{C}^{n-s}$ is an orthogonal mapping,

(b) $F_{\nu} \rightarrow \mathrm{id}_{\mathbb{C}^{n}}$,

(c) $F_{\nu}\left(H_{1}\right)$ is transversal to the submanifold $M$ at the point 0 .

Lemma 2.1 gives $F_{\nu}\left(H_{1}\right) \rightarrow H_{1}$. By Theorem 3.3 we can find a $\nu_{1}$ such that $F_{\nu}\left(H_{1}\right) \in \mathcal{J}_{1}\left(\mathcal{H}, E^{n}, Z\right)$ for $\nu \geq \nu_{1}$. We put $\widetilde{H}_{1}:=F_{\nu_{1}}\left(H_{1}\right), \mathcal{H}^{1}:=\mathcal{H}_{1}\left(\widetilde{H}_{1}\right)$. Obviously there exists $\varepsilon_{1}>0$ such that $\left.\mathcal{H}^{1}\right|_{\varepsilon_{1} E^{n}} \in \mathcal{H}\left(\varepsilon_{1} E^{n}, Z\right)$.

Let $q$ be an integer, $2 \leq q \leq m-s$. Suppose that we have found a system $\mathcal{H}^{q-1}:=\left(\widetilde{H}_{1}, \ldots, \widetilde{H}_{q-1}, \bar{H}_{q}, \ldots, H_{n-s}\right)$ which satisfies the conditions: 
- $\operatorname{dim}\left(\bigcap_{j=1}^{q-1} T_{0} \widetilde{H}_{j} \cap M\right)=m-q+1$,

- there exists an $\varepsilon_{q-1}>0$ such that $\left.\mathcal{H}^{q-1}\right|_{\varepsilon_{q-1} E^{n}} \in \mathcal{H}\left(\varepsilon_{q-1} E^{n}, Z\right)$,

- $\widetilde{\nu}\left(\mathcal{H}^{q-1} \cdot Z, 0\right)=\widetilde{g}(Z, S)(0)$.

Construction of $\widetilde{H}^{q}$. If $H_{q}$ is transversal to $\bigcap_{j=1}^{q-1} \widetilde{H}_{j} \cap M$ at 0 , we put $\widetilde{H}_{q}:=H_{q}$. Otherwise we apply Lemma 2.1 and Theorem 3.3 to find $\widetilde{H}_{q} \in$ $\mathcal{J}_{q}\left(\mathcal{H}^{q-1}, E^{n}, Z\right)$ that is transversal to $\bigcap_{j=1}^{q-1} \widetilde{H}_{j} \cap M$ at 0 . We define $\mathcal{H}^{q}:=$ $\left(\mathcal{H}^{q-1}\right)_{q}\left(\widetilde{H}_{q}\right)$. One can see that there exists an $\varepsilon_{q}>0$ such that $\left.\mathcal{H}^{q}\right|_{\varepsilon_{q} E^{n}} \in$ $\mathcal{H}\left(\varepsilon_{q} E^{n}, Z\right)$.

By Lemma 4.2,

$$
\widetilde{g}_{M}(Z, S)(c) \leq_{\text {lex }} \widetilde{\nu}\left(\mathcal{H}^{m-s} \cdot Z, c\right)=\widetilde{g}_{N}(Z, S)(c) .
$$

Assume that a system $\mathcal{H} \in \mathcal{H}(M, Z)$ satisfies $\widetilde{\nu}(\mathcal{H} \cdot Z, c)=\widetilde{g}_{M}(Z, S)(c)$. Lemma 4.1 yields $\widetilde{g}_{M}(Z, S)(c) \geq_{\text {lex }} \widetilde{g}_{N}(Z, S)(c)$.

5. Intersections on analytic spaces. Let $M$ be a submanifold of a manifold $N$. If $A_{1}, \ldots, A_{r}$ are analytic cycles on $M$, we denote by $A_{1} \bullet M$ $\ldots \bullet M A_{r}$ (resp. $A_{1} \bullet \bullet_{N} \ldots \bullet_{N} A_{r}$ ) their intersection product in $M$ (resp. $N)$. In this section we prove that those cycles are equal and construct the intersection product of analytic cycles on a reduced analytic space.

We will need the following lemmas about systems of hypersurfaces.

Lemma 5.1. Let $N=E^{r n}, M=E^{r m} \times\{0\}^{r n-r m}, S=E^{m} \times\{0\}^{r n-m}$, $S^{\prime}=E^{m} \times\{0\}^{(r-1) n} \times E^{n-m}$, where $r \geq 2$, and let $Z$ be a purely $k$ dimensional analytic subset of $M$. If $\mathcal{H}=\left(H_{1}, \ldots, H_{(r-1) m}\right) \in \mathcal{H}_{S}(M, Z)$, then there exists a system of hypersurfaces $H_{(r-1) m+1}, \ldots, H_{(r-1) n}$ such that (2) $\hat{\mathcal{H}}:=\left(H_{1} \times E^{r n-r m}, \ldots, H_{(r-1) m} \times E^{r n-r m}, H_{(r-1) m+1}, \ldots, H_{(r-1) n}\right)$ belongs to $\mathcal{H}_{S^{\prime}}(N, Z)$ and $\widetilde{\nu}(\widehat{\mathcal{H}} \cdot Z, 0)=(\underbrace{0, \ldots, 0}_{n-m}, \widetilde{\nu}(\mathcal{H} \cdot Z, 0))$.

Proof. Put $H_{j}:=V\left(x_{j}\right) \cap E^{n}$ for $i=(r-1) m+1, \ldots,(r-1) n$ and define $\widehat{\mathcal{H}}$ by (2). The inclusion $\bigcap_{j=1}^{(r-1) m} H_{j} \cap Z \subset S^{\prime}$ implies that $\widehat{\mathcal{H}} \in \mathcal{H}_{S^{\prime}}(N, Z)$, The same inclusion shows that the cycles $\widehat{Z}_{j}^{S}$ vanish for $j>m$, where $\widehat{Z}_{j}$, $\widehat{Z}_{j}^{S^{\prime}}$ (resp. $Z_{j}, Z_{j}^{S}$ ) denote the result of the $j$ th step of the Algorithm applied to $\widehat{\mathcal{H}}$ (resp. $\mathcal{H}$ ), $Z$ and $S^{\prime}$ (resp. $S$ ). The equality $S^{\prime} \cap M=S$ yields $\widehat{Z}_{j}^{S}=\widehat{Z}_{j}^{S^{\prime}}$ for $j \leq(r-1) m$. Therefore it suffices to prove that $Z_{j}=\widehat{Z}_{j}$ for $j \leq(r-1) m$.

It is obvious that the cycles $Z_{0}, \widehat{Z}_{0}$ are equal. Suppose that $Z_{j-1}=\widehat{Z}_{j-1}$. Then $\left[\mathrm{TW}_{2}\right]$, Thm. 2.2, gives $Z_{j}=\widehat{Z}_{j}$ (see the proof of Lemma 4.1).

Lemma 5.2. Under the assumptions of the previous lemma, if $\widehat{\mathcal{H}}=$ $\left(H_{1}, \ldots, H_{(r-1) n}\right)$ belongs to $\mathcal{H}_{S^{\prime}}(M, Z)$, then there exist hypersurfaces 
$H_{(r-1) n+1}, \ldots, H_{r n-m}$ such that $\mathcal{H}:=\left(H_{1}, \ldots, H_{r n-m}\right) \in \mathcal{H}_{S}(N, Z)$ and

$$
(\underbrace{0, \ldots, 0}_{n-m}, \widetilde{\nu}(\mathcal{H} \cdot Z, 0))=\widetilde{\nu}(\widehat{\mathcal{H}} \cdot Z, 0) .
$$

Proof. Put $H_{j}:=V\left(x_{m+j}\right) \cap E^{r n}$ for $j=(r-1) n+1, \ldots, r n-m$ and $\mathcal{H}:=\left(H_{1}, \ldots, H_{r n-m}\right)$. The inclusion $\bigcap_{j=1}^{(r-1) n} H_{j} \cap Z \subset S$ implies that $\mathcal{H} \in \mathcal{H}_{S}(N, Z)$. Let $Z_{j}, Z_{j}^{S}$ (resp. $\widehat{Z}_{j}, \widehat{Z}_{j}^{S}$ ) denote the result of the $j$ th step of the Algorithm applied to $\mathcal{H}$ (resp. $\widehat{\mathcal{H}}$ ), $Z$ and $S$ (resp. $S^{\prime}$ ). By the same inclusion the cycles $\widehat{Z}_{j}^{S}$ vanish for $j>m$. It is obvious that $Z_{j}^{S}=\widehat{Z}_{j}^{S^{\prime}}$ for $j \leq m$.

Given a system of analytic sets $X_{1}, \ldots, X_{r} \subset M$ of pure dimension and a point $a \in M$, we define the integer

$$
d_{M}\left(X_{1}, \ldots, X_{r}\right)(a):=g_{M^{r}}\left(X_{1} \times X_{r}, \Delta_{M}\right)\left((a)^{r}\right),
$$

where $\Delta_{M}$ denotes the diagonal in $M^{r}$.

Proposition 5.3. Let $M$ be an $m$-dimensional submanifold of a manifold $N, \operatorname{dim} N=n, a \in M$, and let $X_{1}, \ldots, X_{r}$ be analytic subsets of $M$ of pure dimension. Then

$$
\begin{gathered}
(\underbrace{0, \ldots, 0}_{n-m}, \widetilde{g}_{M^{r}}\left(X_{1} \times \ldots \times X_{r}, \Delta_{M}\right)\left((a)^{r}\right)=\widetilde{g}_{N^{r}}\left(X_{1} \times \ldots \times X_{r}, \Delta_{N}\right)\left((a)^{r}\right), \\
d_{M}\left(X_{1}, \ldots, X_{r}\right)(a)=d_{N}\left(X_{1}, \ldots, X_{r}\right)(a) .
\end{gathered}
$$

Proof. It suffices to prove the first equality. Lemma 5.2 yields $\left(0, \ldots, 0, \widetilde{g}_{N^{r}}\left(X_{1} \times \ldots \times X_{r}, \Delta_{M}\right)\left((a)^{r}\right) \leq_{\text {lex }} \widetilde{g}_{N^{r}}\left(X_{1} \times \ldots \times X_{r}, \Delta_{N}\right)\left((a)^{r}\right)\right.$. By Lemma 5.1,

$(\underbrace{0, \ldots, 0}_{n-m}, \widetilde{g}_{M^{r}}\left(X_{1} \times \ldots \times X_{r}, \Delta_{M}\right)\left((a)^{r}\right) \geq \operatorname{lex} \widetilde{g}_{N^{r}}\left(X_{1} \times \ldots \times X_{r}, \Delta_{N}\right)\left((a)^{r}\right)$.

Theorem 4.3 concludes the proof.

As an immediate consequence we obtain the following theorem.

THeOREM 5.4. Let $M$ be a submanifold of a manifold $N$, and let $A_{1}, \ldots$ $\ldots, A_{r}$ be analytic cycles on $M$. Then

$$
A_{1} \bullet \bullet_{N} \ldots \bullet_{N} A_{r}=A_{1} \bullet_{M} \ldots \bullet_{M} A_{r} .
$$

Let $W$ be a reduced analytic space. We set

$\mathcal{K}(W):=\{f: W \rightarrow \mathbb{C}: f(W) \subset \mathbb{Z}, f$ is analytically constructible $\}$, and let $\mathcal{G}(W)$ denote the family of analytic cycles on $W$.

We have the following generalization of $\left[\mathrm{T}_{2}\right]$, Prop. 2.1.3. 
Lemma 5.5. The mapping $\nu: \mathcal{G}(W) \ni A \mapsto \nu(A) \in \mathcal{K}(W)$ is a bijection.

Proof. Obviously $\nu$ is an additive injection. Fix $f \in \mathcal{K}(W)$. Our aim is to construct a cycle $T$ satisfying $\nu(T)=f$.

Put $W_{0}:=W, f_{0}:=f$. Let $\left\{W_{0}^{j}\right\}_{j \in J}$ be the irreducible components of the space $W$. Since the proof of $\left[\mathrm{T}_{2}\right]$, Prop. 2.1.3, is valid for irreducible analytic spaces, we can find a family $\left\{T_{0}^{j}\right\}_{j \in J}$ of analytic cycles such that $T_{0}^{j} \in \mathcal{G}\left(W_{0}^{j}\right)$ and $\nu\left(T_{0}^{j}\right)=\left.f\right|_{W_{0}^{j}}$. Observe that $T_{0}:=\sum_{j \in J} T_{0}^{j}$ is an analytic cycle and define $W_{1}:=\operatorname{sing} W_{0}$ and $f_{1}:=f_{0}-\nu\left(T_{0}\right)$. Since $\operatorname{supp}\left(f_{1}\right) \subset W_{1}$, we can decompose $W_{1}$ and construct the cycle $T_{1}$.

Repeating this procedure we obtain a sequence $\left\{W_{j}, T_{j}, f_{j}\right\}_{j=0}^{\infty}$, where $T_{j} \in \mathcal{G}\left(W_{j}\right), f_{j} \in \mathcal{K}\left(W_{j}\right), W_{j}$ are analytic subsets of $W$, which satisfies the following conditions:
$\left(\mathrm{a}_{i}\right)$
$\left(\mathrm{b}_{i}\right)$$$
f_{i}=f_{i-1}-\nu\left(T_{i-1}\right),
$$$$
\operatorname{supp}\left(f_{i}\right) \subset W_{i},
$$

$$
W_{i}=\operatorname{sing} W_{i-1} \text {. }
$$

The condition $\left(a_{i}\right)$ yields

$$
\sum_{j=0}^{i} f_{j}-\sum_{j=0}^{i} \nu\left(T_{j}\right)=\sum_{j=1}^{i+1} f_{j} .
$$

By $\left(\mathrm{b}_{i}\right),\left(\mathrm{c}_{i}\right)$ the formal sum $T:=\sum_{j=0}^{\infty} T_{j}$ is a cycle. Fix $x \in W$. By the same conditions there exists a $j_{0}$ such that the functions $f_{j}, \nu\left(T_{j}\right)$ vanish at $x$ for $j \geq j_{0}$. The equality $\left(\mathrm{d}_{i}\right)$ yields $f(x)-\nu(T, x)=0$.

As an immediate consequence of Proposition 5.3 we get the following lemma.

LEMma 5.6. Let $W$ be a reduced analytic space, $X_{1}, \ldots, X_{r} \subset W$ be analytic sets of pure dimension, and $a \in W$. Let $\psi_{i}: U_{i} \rightarrow Z_{i}$ be charts for $i=1,2$, where $U_{i}$ is a neighbourhood of $a$ and $Z_{i}$ is an analytic subset of a complex manifold $M_{i}$. Then

$$
d_{M_{1}}\left(\psi_{1}\left(X_{1}\right), \ldots, \psi_{1}\left(X_{r}\right)\right)\left(\psi_{1}(a)\right)=d_{M_{2}}\left(\psi_{2}\left(X_{1}\right), \ldots, \psi_{2}\left(X_{r}\right)\right)\left(\psi_{2}(a)\right) .
$$

Now we define the multiplicity of intersection of sets $X_{1}, \ldots, X_{r}$ at a point.

Definition 5.7. Let $W$ be a reduced analytic space, $X_{1}, \ldots, X_{r}$ be its irreducible analytic subsets, and $a \in W$. Let $\psi: U \rightarrow Z$ be a chart, where $U$ is a neighbourhood of $a$ and $Z$ is an analytic subset of a complex manifold $M$. The multiplicity of intersection of $X_{1}, \ldots, X_{r}$ at the point $a$ is defined to be

$$
d\left(X_{1}, \ldots, X_{r}\right)(a):=d_{M}\left(\psi\left(X_{1}\right), \ldots, \psi\left(X_{r}\right)\right)(\psi(a)) .
$$


By $\left[\mathrm{T}_{2}\right]$, Thm. 5.5, and Lemma 5.6, the function $W \ni x \mapsto d\left(X_{1}, \ldots\right.$ $\left.\ldots, X_{r}\right)(x)$ is analytically constructible.

DeFinition 5.8. Let $X_{1}, \ldots, X_{r}$ be irreducible analytic subsets of a reduced analytic space $W$. Their intersection product $X_{1} \bullet \ldots \bullet X_{r}$ is defined to be the unique cycle such that $\nu\left(X_{1} \bullet \ldots \bullet X_{r}\right)=d\left(X_{1}, \ldots, X_{r}\right)$.

As in the smooth case (see $\left[\mathrm{T}_{2}\right]$, Def. 6.4) we extend this definition by $\mathbb{Z}$-linearity to the case of arbitrary analytic cycles on a reduced analytic space.

6. Regular separation on analytic spaces. Let $W$ be a reduced analytic space. In this section we study the relation between intersection multiplicity and separation exponent for analytic subsets of $W$. By $[\mathrm{Cg}]$, Cor. 4.5, if $W$ is smooth, then $X$ and $Y$ are $p$-separated at $a \in X \cap Y$ with $p=\nu\left(X \bullet_{W} Y, a\right)$. We will prove that the same holds in the case of analytic subsets of a reduced analytic space.

We begin by recalling some basic facts (see $\left[\mathrm{T}_{1}\right],[\mathrm{Cg}],[\mathrm{CT}]$ for details). Let $X, Y$ be locally analytic subsets of a normed complex vector space $M$. Let $|\cdot|$ denote the norm in $M$ and let $\varrho(\cdot, Z)$ stand for the distance to the set $Z \subset M$. For $p \in[1, \infty)$ we say that $X, Y$ are $p$-separated at a point $a \in X \cap Y$ if there exist $C, r>0$ such that

$$
\varrho(z, X)+\varrho(z, Y) \geq C \varrho(z, X \cap Y)^{p}
$$

provided $|z-a|<r$. We generalize the above definition in the following way.

DeFinition 6.1. Let $X, Y$ be analytic subsets of the space $W$. We say that $X$ and $Y$ are $p$-separated at $a \in X \cap Y$ if for every chart $\varphi: V \rightarrow G$, where $V$ is a neighbourhood of $a$, the sets $\varphi(X), \varphi(Y)$ are $p$-separated at $\varphi(a)$.

LEMMA 6.2. Let $X, Y$ be analytic subsets of the space $W$, and $a \in X \cap Y$. If $\psi: U \rightarrow G$, where $U$ is a neighbourhood of $a$, is a chart, and $p \in[1, \infty)$, then the following conditions are equivalent:

(1) $X$ and $Y$ are $p$-separated at $a$,

(2) the sets $\psi(X), \psi(Y)$ are $p$-separated at $\psi(a)$.

Proof. Let $k<n$ be positive integers. It suffices to prove that if $X$ and $Y$ are analytic subsets of $E^{k}$, then the following conditions are equivalent:

(a) $X$ and $Y$ are $p$-separated at 0 (in $E^{k}$ ),

(b) $X \times\{0\}^{n-k}$ and $Y \times\{0\}^{n-k}$ are $p$-separated at 0 (as subsets of $E^{n}$ ). Obviously (b) implies (a). To prove the other implication consider $\mathbb{C}^{n}$ with the euclidean norm and apply [Cg], Lemma 4.2.

As an immediate consequence of $[\mathrm{Cg}]$, Cor. 4.5, we obtain the following theorem. 
THEOREM 6.3. Let $X, Y$ be analytic subsets of a reduced analytic space $W$ and let $a \in X \cap Y$. Then $X$ and $Y$ are $p$-separated at $a$ with $p=\nu(X \bullet Y, a)$.

Acknowledgements. I would like to express my sincere thanks to Piotr Tworzewski for suggesting the subject and many helpful conversations.

\section{References}

[AM] R. Achilles and M. Manaresi, Multiplicities of a bigraded ring and intersection theory, Math. Ann. 309 (1997), 573-591.

[ATW] R. Achilles, P. Tworzewski and T. Winiarski, On improper isolated intersection in complex analytic geometry, Ann. Polon. Math. 51 (1990), 21-36.

[BH] A. Borel et A. Haefliger, La classe d'homologie fondamentale d'un espace analytique, Bull. Soc. Math. France 89 (1961), 461-513.

$[\mathrm{Cg}]$ E. Cygan, Intersection theory and separation exponent in complex analytic geometry, Ann. Polon. Math. 69 (1998), 287-299.

[CKT] E. Cygan, T. Krasiński and P. Tworzewski, Separation at infinity and Lojasiewicz exponent of polynomial mappings, Invent. Math. 136 (1999), 75-87.

[CT] E. Cygan and P. Tworzewski, Proper intersection multiplicity and regular separation of analytic sets, Ann. Polon. Math. 59 (1994), 293-298.

[Dr] R. N. Draper, Intersection theory in analytic geometry, Math. Ann. 180 (1969), $175-204$.

$\left[\mathrm{R}_{1}\right]$ S. Rams, Convergence of holomorphic chains, Ann. Polon. Math. 65 (1997), $227-234$.

$\left[\mathrm{R}_{2}\right]$-, Bézout-type theorems for certain analytic sets, Bull. Polish Acad. Sci. Math. 46 (1998), 277-283.

$\left[\mathrm{T}_{1}\right]$ P. Tworzewski, Isolated intersection multiplicity and regular separation of analytic sets, Ann. Polon. Math. 58 (1993), 213-219.

$\left[\mathrm{T}_{2}\right]$-, Intersection theory in complex analytic geometry, Ann. Polon. Math. 62 (1995), 177-191.

$\left[\mathrm{TW}_{1}\right]$ P. Tworzewski and T. Winiarski, Continuity of intersection of analytic sets, Ann. Polon. Math. 42 (1983), 387-393.

$\left[\mathrm{TW}_{2}\right]-,-$, Cycles of zeros of holomorphic mappings, Bull. Polish Acad. Sci. Math. 37 (1989), 95-101.

Institute of Mathematics

Jagiellonian University

Reymonta 4

30-059 Kraków, Poland

E-mail: rams@im.uj.edu.pl 